\title{
Posterior Reversible Encephalopathy Syndrome (PRES) Secondary to Severe Pneumonia
}

\author{
Solangaarachchige Anushika Luckmy ${ }^{1^{*}}$, Kumanan Thirunavukarasu ${ }^{1}$, Gowry Selvaratnam ${ }^{1}$ and Muthusamy Malaravan ${ }^{2}$ \\ ${ }^{1}$ University Medical Unit, Jaffna Teaching Hospital, Hospital Road, Jaffna, Sri Lanka \\ ${ }^{2}$ Department of Ophthalmology, Jaffna Teaching Hospital, Hospital Road, Jaffna, Sri Lanka
}

*Corresponding author: Solangaarachchige AL, University Medical Unit, Jaffna Teaching Hospital, Hospital Road, Jaffna, Sri Lanka, Tel: +940779519435; E-mail: saluckmy@gmail.com

Received Date: Sep 12, 2017; Accepted Date: Nov 09, 2017; Published Date: Nov 20, 2017

Copyright: (c) 2017 Luckmy SA, et al. This is an open-access article distributed under the terms of the Creative Commons Attribution License, which permits unrestricted use, distribution, and reproduction in any medium, provided the original author and source are credited.

\begin{abstract}
Posterior reversible encephalopathy is a rare neurological disease. The association between Posterior reversible encephalopathy syndrome and severe pneumonia is not well described in the available medical literature. We describe a case of a 51 year old female presented to our hospital with sudden loss of vision in both eyes in the background history of pneumonia who recovered completely with treatment. This case report elaborates the very rare entity of posterior reversible encephalopathy syndrome secondary to severe pneumonia.
\end{abstract}

Keywords: Neurological manifestations; Posterior reversible encephalopathy syndrome; PRES; Severe pneumonia

\section{Introduction}

Posterior reversible encephalopathy syndrome is also referred as PRES. PRES was first described in the medical literature in 1996. It is a distinct clinical condition, but considered as a stroke like syndrome due to sharing some of the clinical features of stroke [1,2]. PRES can be resulted due to many conditions including acute hypertension, autoimmune conditions, drugs, chronic inflammatory conditions and severe infections [1]. Usually PRES has an excellent outcome once the causative factor is controlled. Neurological involvement of pneumonia is already known fact. Especially atypical pneumonia caused by Mycoplasma pneumonia and Legionella peumophila are more potential to associate with classic neurological manifestations such as meningoencephalitis, aseptic meningitis, myelitis, cranial nerve palsies. But severe pneumonia causes PRES as a neurological sequalae is a very rare association [3].

\section{Case Presentation}

A 51 year old female presented with sudden loss of vision in both eyes for 4 hours. No pain or redness of the eyes was reported. She had a five day duration of febrile illness with cough, nasal stuffiness and runny nose prior to this presentation. She was treated by a general practitioner with oral azithromycin $500 \mathrm{mg}$ once a day for 3 days. On the fifth day of the illness, she reported loss of vision by anecdotal evidence stated by her husband. It was mentioned that she was not able to see anything around $9 \mathrm{am}$ in a well lit room when she tried to move around in the house. The situation did not improve when she was assisted to be outdoors. Interestingly, her primary complaint at the moment was weakness rather than loss of vision as she sought for mobilizing assistance inspire of absence of limb weakness or unsteadiness. Her speech was slurred. She did not experience any similar events in the past. She denied headache, giddiness, vertigo or vomiting. Her consciousness was preserved and oriented. She had no seizures. No preceding chest pain or palpitation was noted. She could not recall any trauma to head and neck. She did not suffer from arthalgia, rashes or oral ulcers. She didn't complain any constitutional symptoms like anorexia, malaise or weight loss. No significant bleeding tendency was noted in the history. Her first pregnancy was uneventful. She had pregnancy induced hypertension during her second pregnancy 7 years back. She had undergone 2 caesarean sections at 37 and 36 weeks during her two pregnancies respectively. She took antihypertensive medications until 2 years following the birth of the second child. She did not follow any outpatient clinics thereafter. Family history was not significant except the fact that her mother had a stroke at the age of 63 years.

Her physical examination indicated Glasgow coma scale score (GCS) was 15/15. She was mildly confused and afebrile on admission. Visual acuity was limited to the perception of light in both eyes (PLboth eyes). Visual field could not be tested. She had preserved direct and consensual light reflexes. Accommodation reflex was unable to perform. Fundoscopy revealed a normal retina, discs and a vascular appearance. Other cranial nerves were clinically intact.

Motor system and sensory system examination revealed normal findings. She was tachycardic with pulse rate of $116 \mathrm{bpm}$ and regular in rhythm. Blood pressure was recorded as $180 / 100 \mathrm{mmHg}$. There were no murmurs or bruits heard on auscultation. Respiratory rate was 22 breath cycles per minute. Her oxygen saturation in ambient air was $73 \%$ and $85 \%$ with 5 litres of supplementary oxygen. Lung auscultation revealed vesicular breathing with bilateral crackles (more prominent in left side) and rhonchi.

Non contrast Computer tomography (CT) brain was done on admission as shown in Figure 1. It showed hypodensed areas in both occipital lobes and right posterior temporal lobes suggestive of an infarction. CT venogram concluded the absence of cerebral venous sinus thrombosis. Magnetic resonance imaging (MRI) of brain was done after 2 weeks and it revealed normal bilateral occipital lobes which supported the diagnosis of PRES. 
Citation: Luckmy SA, Thirunavukarasu K, Selvaratnam G, Malaravan M (2017) Posterior Reversible Encephalopathy Syndrome (PRES) Secondary to Severe Pneumonia. Med Rep Case Stud 2: 144. doi:10.4172/2572-5130.1000144

Page 2 of 4

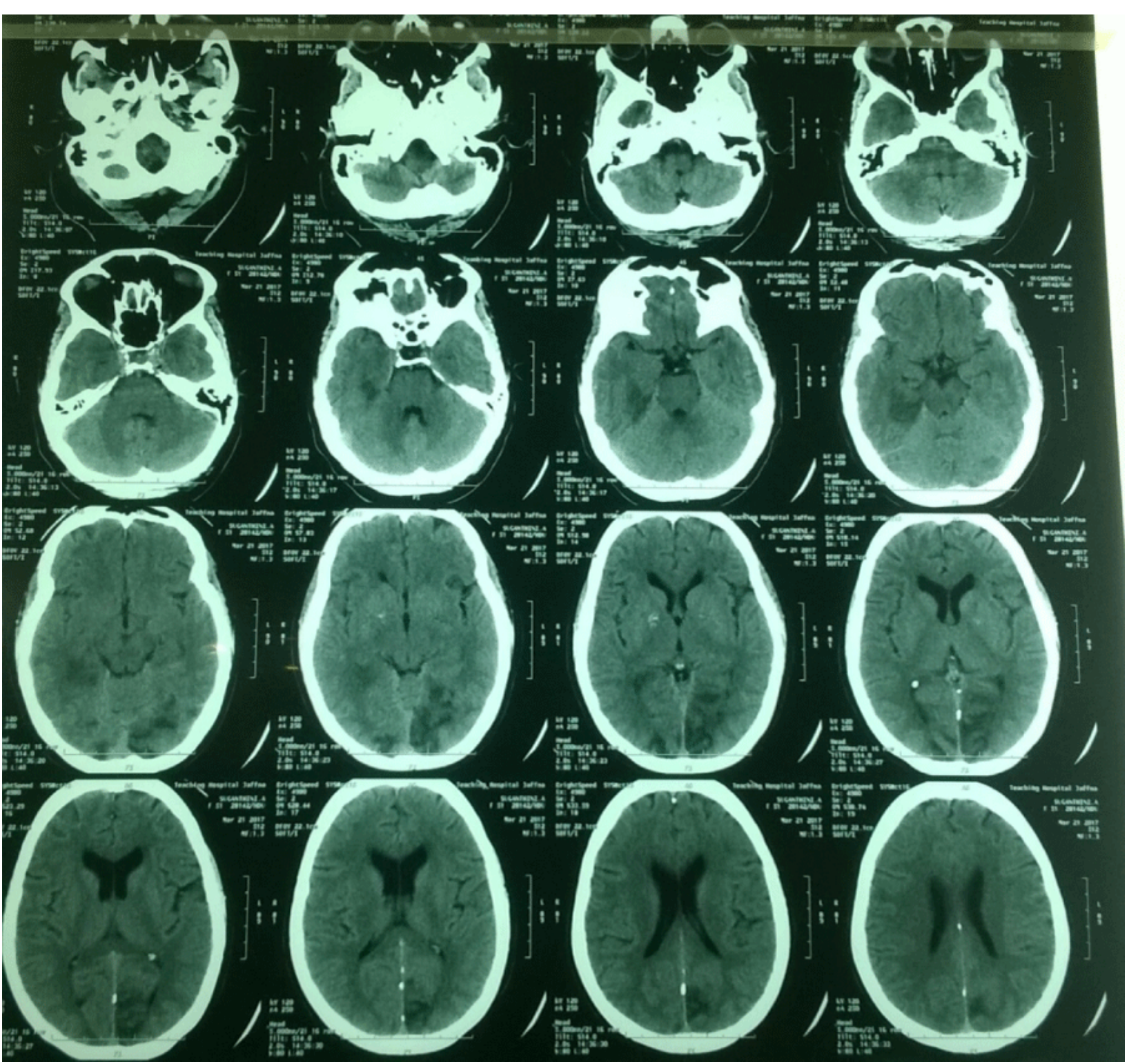

Figure 1: NCCT brain scan which was done on admission illustrated hypodensed areas in bilateral occipital lobes and right posterior temporal lobes.

She had been evaluated for possible thromboembolism. Electrocardiogram recorded a sinus rhythm and no significant left ventricular hypertrophy noted. Cardiac sources of thromboembolism were excluded by a $2 \mathrm{D}$ echocardiogram. Her clotting profile was within the normal limits. Arterial blood gas analysis was consistent with hypoxaemic respiratory failure. Arterial blood gas analysis showed $\mathrm{pH}$ 7.38 (7.35-7.45), $\mathrm{pCO}_{3} 29 \mathrm{mmHg}(35-45), \mathrm{pO}_{2} 60 \mathrm{mmHg}(80-100)$, $\mathrm{HCO}_{3} 19.8 \mathrm{mEq} / \mathrm{l}(22-26), \mathrm{BE}-7.9$. Complete blood count was in the normal limits including white blood cells $8.65 \times 10^{9} / 1$ (4.00-10.00), neutrophils $63.1 \%$ (40-80), lymphocytes $23.5 \%$ (20-40), monocytes 9.4\% (2-10), eosinophils $3.5 \%$ (1-6), basophils $0.5 \% \quad(0.0-2)$, haemoglobin level $13.7 \mathrm{~g} / \mathrm{dl}$ (13-16), platelets $275 \times 10^{9} / \mathrm{l}(150-410)$. C reactive protein level was $19.2 \mathrm{mg} / \mathrm{dl}(<6)$ and ESR $65 \mathrm{~mm} /$ first hour. Blood was negative for influenza antigen in reverse transcription polymerase chain reaction. Antinuclear factor was within the normal range.

Clinical diagnosis of Posterior reversible encephalopathy syndrome (PRES) was made. Patient's high blood pressure was treated with oral losartan $50 \mathrm{mg}$ twice a day. On the following day patient became more dyspnoeic and developed respiratory distress. Patient was admitted to the intensive care unit and she did not require endotracheal intubation and ventilation. She was given oral osetalmivir $75 \mathrm{mg}$ twice a day for 5 days and intravenous ceftazidime $1 \mathrm{~g}$ twice a day for 10 days according to the sensitive pattern of sputum culture which was positive for Pseudomonas species. Her visual loss slowly improved and visual assessment was performed periodically. Patient was continued to be tachycardic and had persistent high blood pressure which prompted us to upgrade antihypertensives with oral hydrochlothiazide $25 \mathrm{mg}$ once a 
day and oral nifedipine $20 \mathrm{mg}$ twice a day. Over the next few days, patient's clinical condition was improved gradually as shown in Figures 2 and 3. She was discharged home on the 16th day of the illness and her corrected visual acuity in both eyes $6 / 24$ with N14 near vision and had a right side homonymous hemianopia. Chest X-ray showed a resolution of inflammatory shadows.

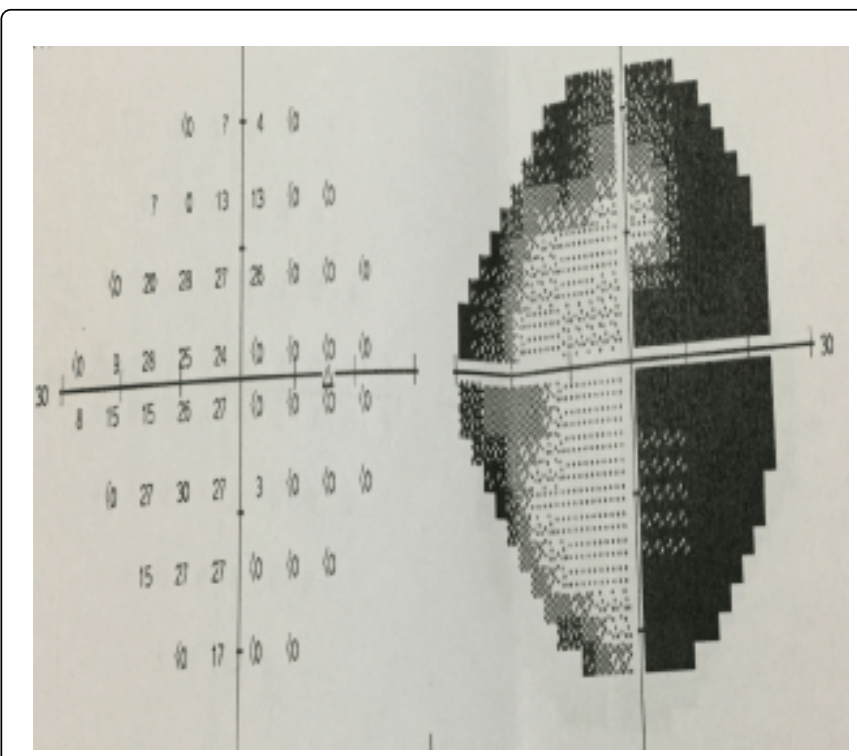

Figure 2: Right side visual field of the patient two weeks after admission.

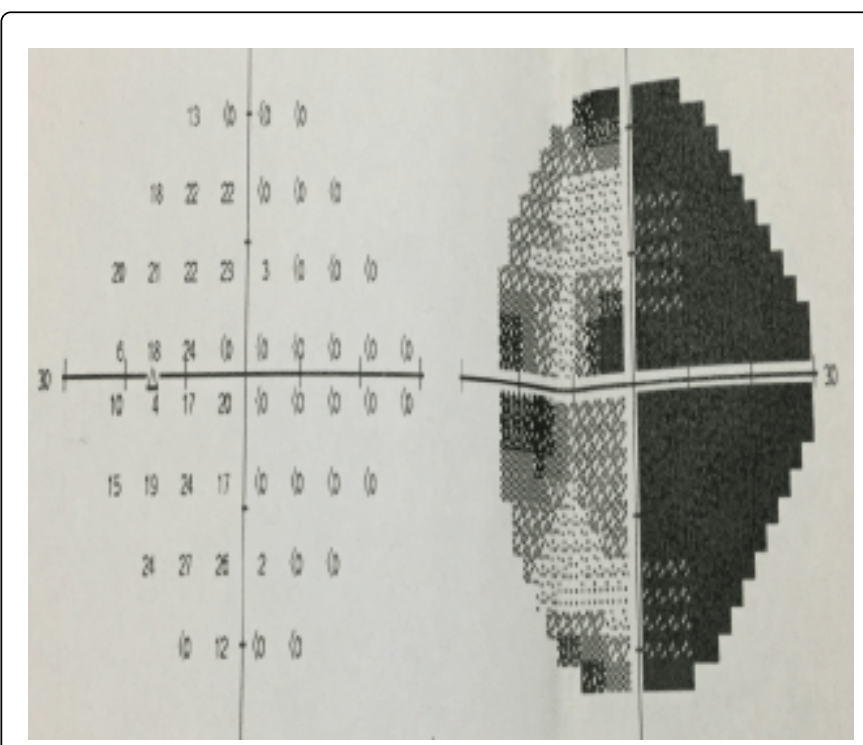

Figure 3: Left side visual field of the patient two weeks after admission.

\section{Discussion}

PRES is a relatively new terminology in the field of medicine as it was first described in 1996. PRES is increasingly recognized in the current practice which is believed to be due to the evolution of modern neuroimaging facilities $[4,5]$. During the last two decades, MRI scan has allowed for increased number of case detection in PRES [6]. Common MRI findings of PRES are bilaterally symmetrical subcortical or cortical hyperintensity in T2 weighted images and the evidence of vasogenic oedema [4,6]. Among the reported case of PRES, its common presentations included confusion, seizures, visual impairment, headache, vomiting [2]. However PRES is a complex clinical condition which is not fully understood yet. PRES has been noted to have a good clinical outcome. Thus PRES has numerous associations; it has been proved that acute hypertension has an immense impact on PRES [6]. Toxins, drugs, eclampsia, autoimmune diseases, malignancies are also known as causative factors [1,2,7]. Although there have been reported cases with PRES and severe infection and sepsis, there are no definitive cases found in the literature in order to severe pneumonia and PRES [7]. Infection related PRES was $8-24 \%$. When PRES is associated with sepsis, it is more likely to be due to gram positive bacteria preferably by gram positive cocci [7]. We assume that PRES in a case with infection caused by gram negative bacteria is very rare. Mycoplasma pneumoniae, Legionella pneumophila are the commonest bacteria which are more capable of causing numerous extra pulmonary manifestations included neurological syndromes such as meningoencephalitis, aseptic meningitis, cerebellitis, cranial nerve palsies, polyradiculopathy [3]. But severe pneumonia causes PRES as a neurological consequence is not found in the current medical literature. Neurological manifestations of pneumonia are frequently categorized under two groups such as neurological symptoms which occur parallel with respiratory symptoms and neurological symptoms which appear later following a gap [3]. Mechanisms of underling PRES in pneumonia and sepsis have not yet been elucidated [4,7]. But there are some theories which have been used to explain this association. The postulated theory of infection, sepsis, shock and associated PRES would also explain the pathogenesis of pneumonia and PRES. The pathophysiology of PRES is multifactorial in sepsis. Body response to any bacterial infection is mediated by both exotoxins and endotoxins $[1,7]$. These chemical mediators are also strong stimulants to initiate an inflammatory response. Vascular instability, direct invasion, neurotoxicity are complex mechanisms of pathophysiology in PRES in sepsis. Endothelin 1 is one of the very potent vasoconstrictor which also plays a key role [6].

This case had a severe pneumonia and the diagnosis is supported by clinical findings, hypoxaemic respiratory failure, extensive chest X-ray shadows, high inflammatory markers. Antibiotic treatment prior to admission would have resulted normal complete blood count. This patient presented during the period of seasonal influenza infection was its peak. But it could not be proved as influenza antigen became negative. However the underlying untreated hypertension also thought to be contributed to PRES in this case or may be coincidential.

According to the MRI scan of brain which was done two weeks following the occurrence of symptoms had a resolution and clinical improvement would strongly suggest the diagnosis of PRES. It took 2 to 3 weeks for the patient's vision to be restored. She followed the medical clinic and attended regular opthalmological assessments. She had a good adherence to treatment too. Four weeks after the initial presentation, she resumed her previous job as a lecturer.

This case prompts clinicians to consider PRES in the clinical practice, even in a case with pneumonia and the importance of early diagnosis. This will be the first case report with PRES in severe pneumonia responded effectively with treatment. Still more evidence is needed in the future in regards to this association. 


\section{References}

1. Roth C, Ferbert A (2011) The posterior reversible encephalopathy syndrome: what's certain, what's new? Pract Neurol 11: 136-144.

2. Hobson EV, Craven I, Blank SC (2012) Posterior Reversible Encephalopathy Syndrome: A Truly Treatable Neurologic Illness. Perit Dial Int 32(6): 590-594.

3. Panagariya A, Sharma AK, Dev A, Kankane A, Sharma B, et al. (2011) Reversible neurological syndromes with atypical pneumonia. Ann Indian Acad Neurol 14(2): 127-129.

4. Bartynski WS (2008) Posterior reversible encephalopathy syndrome, part 2: controversies surrounding pathophysiology of vasogenic edema. AJNR Am J Neuroradiol 29: 1043-1049.
5. Ishihara M, Matsutani N, Ota S, Seki N (2017) A Case of Posterior Reversible Encephalopathy Syndrome Induced by Cisplatin/Pemetrexed Chemotherapy for Lung Cancer. Case Rep Oncol 10(1):235-238.

6. Fischer M, Schmutzhard E (2017) Posterior reversible encephalopathy syndrome. Journal of Neurology 264(8): 1608-1616.

7. Bartynski WS, Boardman JF, Zeigler ZR, Shadduck RK, Lister J (2006) Posterior reversible encephalopathy syndrome in Infection, Sepsis, and Shock. AJNR Am J Neuroradiol 27(10): 2179-2190. 